\title{
Surgical Planning and Patient-Specific Biomechanical Simulation for Tracheal Endoprostheses Interventions
}

\author{
Miguel A. González Ballester ${ }^{1}$, Amaya Pérez del Palomar², \\ José Luís López Villalobos ${ }^{3}$, Laura Lara Rodríguez ${ }^{1}$, Olfa Trabelsi ${ }^{2}$, Frederic Pérez ${ }^{1}$, \\ Ángel Ginel Cañamaque ${ }^{3}$, Emilia Barrot Cortés ${ }^{3}$, Francisco Rodríguez Panadero ${ }^{3}$, \\ Manuel Doblaré Castellano ${ }^{2}$, and Javier Herrero Jover ${ }^{1,4}$ \\ ${ }^{1}$ Alma IT Systems, Barcelona, Spain \\ ${ }^{2}$ Group of Structural Mechanics and Material Modelling, University of Zaragoza, Spain \\ ${ }^{3}$ Medico-Surgical Dept. of Respiratory Diseases, Virgen del Rocío Hospital, Seville, Spain \\ ${ }^{4}$ Centro Médico Teknon, Barcelona, Spain \\ Miguel.Gonzalez@alma3d.com
}

\begin{abstract}
We have developed a system for computer-assisted surgical planning of tracheal surgeries. The system allows to plan the intervention based on CT images of the patient, and includes a virtual database of commercially available prostheses. Automatic segmentation of the trachea and apparent pathological structures is obtained using a modified region growing algorithm. A method for automatic adaptation of a finite element mesh allows to build a patient-specific biomechanical model for simulation of the expected performance of the implant under physiological movement (swallowing, sneezing). Laboratory experiments were performed to characterise the tissues present in the trachea, and movement models were obtained from fluoroscopic images of a patient. Results are reported on the planning and biomechanical simulation of two patients that underwent surgery at our hospital.
\end{abstract}

\section{Introduction}

A number of pathologies affecting the trachea exist, both benign and malignant, leading to the obstruction of the airways and, ultimately, to asphyxia. Tracheostomy consists in performing an orifice in the trachea, distal to the obstruction, to ensure access to the air. This type of procedures - reported as early as 3.600 b.C. - have evolved towards new therapeutic variants, leading to the design of modern tracheal endoprostheses and stents 1 .

Preoperative planning of tracheal surgery is performed nowadays on CT or MR images of the patient. Planning includes the selection of the material, type and placement of the prosthesis, and these choices are largely influenced by the previous experience of the surgeon. Further, planning based on a single static image of the patient does not portray the behaviour of the implant under stress conditions derived from physiological movements (swallowing, sneezing, etc.).

We have developed a system for computer-aided planning and simulation, allowing to explore the anatomy of the patient and automatically segment the trachea and 
any apparent pathological structures. A database of models of commercially available tracheal implants permits to select and virtually position the implant of choice. Finally, the established plan is used as initial condition for biomechanical simulations of swallowing movement. Prior to such simulations, laboratory experiments were performed to characterise the tissues present in the trachea and thus build realistic constitutive models.

We present our framework for surgical planning (section 2) and biomechanical simulation (section 3). Results are presented on data from 2 patients that underwent tracheal surgery with the implantation of an endoprosthesis (section 4).

\section{Surgical Planning}

We have developed a software for surgical planning of tracheal implants. The tool supports full connectivity to PACS systems and DICOM conformance, as well as professional 2D, MPR and 3D volume rendering capabilities for the exploration of the data sets. We have implemented an implant database integrated into the GUI of the application, allowing to explore the full range of implant models and sizes commercially available, and including CAD models of each of them for virtual placement. Intuitive manipulation tools allow to fine tune the selection and placement of the implant, and determine potential complications due to factors such as the distance to the vocal chords and the tracheal wall.

\subsection{Automatic Segmentation of the Trachea}

Several segmentation methods for automatic identification and delineation of the trachea in CT images have been tested, ranging from basic thresholding algorithms

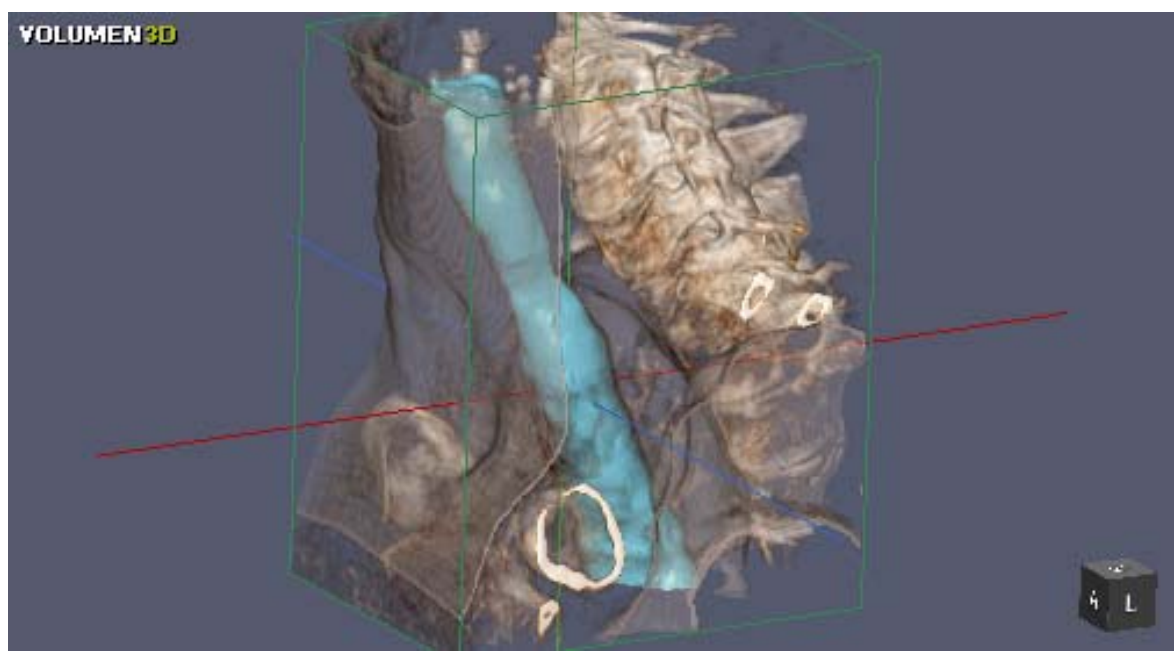

Fig. 1. Automatic segmentation of the trachea (shown in blue) based on anisotropic diffusion, region growing and morphological operations. An alternative approach based on level sets was tested and yielded similar results at higher computational cost. 
to sophisticated level-set evolution techniques 2. Based on criteria such as speed, robustness and minimum user interaction, we designed an adapted region growing method consisting of the following steps:

1) anisotropic diffusion 3 ;

2) adaptive region growing 4 ;

3) morphological operations for segmentation refinement 5 .

Segmentation results were validated by surgeons on 30 cases, via detailed visual inspection. An example of the application of this algorithm to one of our datasets is shown in figure 1 .
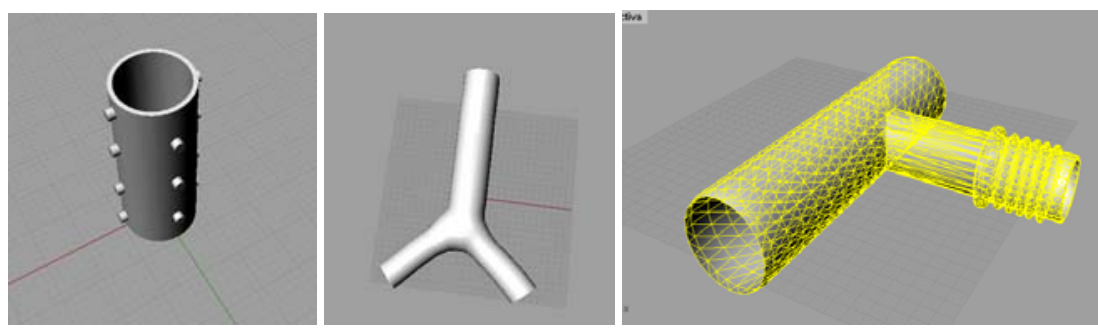

Fig. 2. A database of virtual models of commercially available tracheal endoprostheses was implemented. This allows to make multiple simulations using different implant models and sizes.
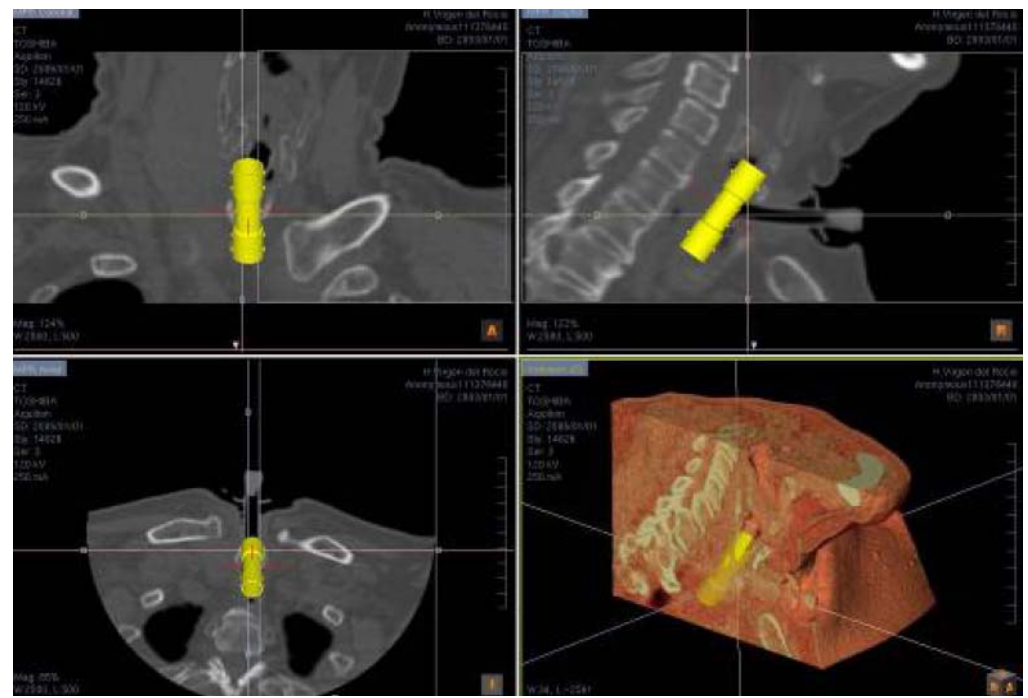

Fig. 3. Planning module for virtual placement of tracheal implants. A preoperative CT scan is used as reference to plan the position and orientation of the chosen implant. The plan is stored and further used for biomechanical simulations. 


\subsection{Implant Database and Virtual Placement}

We have created 3D models of a total of 93 prostheses of different models and sizes (figure 2). These models have been incorporated into our software as a "virtual catalogue". Once a model has been selected, the 3D representation of the implant is shown on the patient's image data. An intuitive 3D interface allows to virtually position and align the prosthesis (figure 3 ).

\section{Biomechanical Simulation}

The choice of implant model, size and position has been made based on a single static CT image of the patient. However, as part of its normal function, the trachea undergoes demanding physiological movements that have an important effect on the performance and lifespan of the prosthesis. To take this into account, we create a patient-specific biomechanical model of the trachea and the implant, and perform finite element simulations to predict the dynamic behaviour and thus identify possible risks due to excessive stress on the tracheal walls.

\subsection{Patient-Specific Models of the Trachea}

We built a detailed finite element mesh including all tissues present in the trachea. This model was constructed from a CT data set of a subject with no tracheal pathology, using the segmentation method described above and the commercial meshing software packages: PATRAN (MSC, Santa Ana, USA) and I-DEAS (Siemens PLM, Plano, USA). The resulting mesh contains 28.350 hexahedral elements, grouped into 3 groups: membrane, tracheal muscle, and cricoid and thyroid cartilage (figure 4, left).
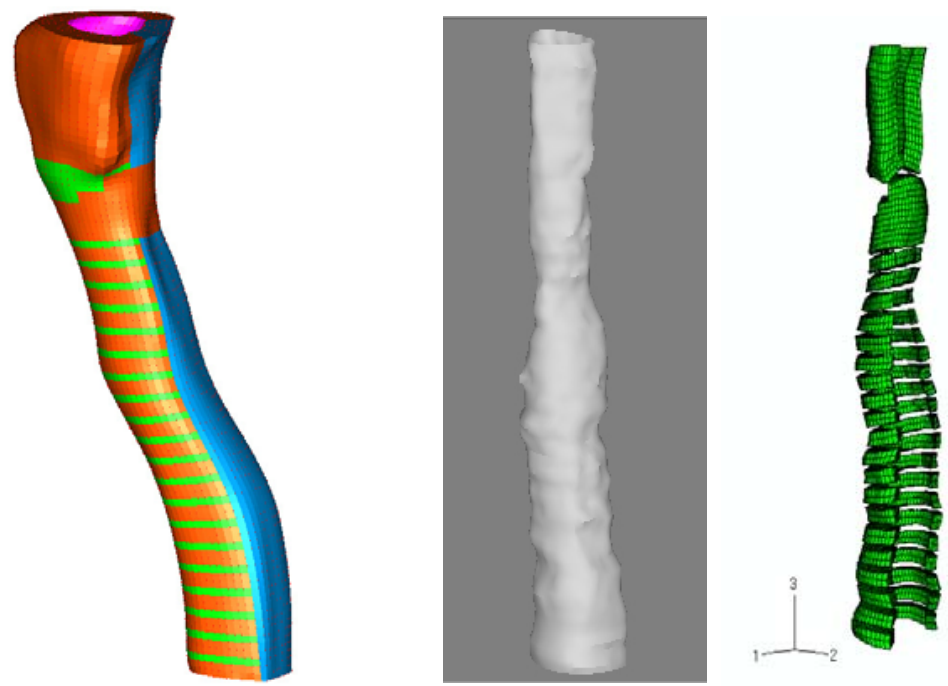

Fig. 4. Complete finite element model of the human trachea, including cartilage (red), muscle (blue), internal membrane (green). On the right, automatic adaptation of the finite element mesh (cartilage is shown) to a new tracheal geometry, in this case with a stenosis, obtained by deforming the mesh to the segmented tracheal surface of a patient (middle). 
This mesh can be adapted to the particular patient's anatomy by deforming it to match the segmented internal and external surfaces of the patient's trachea. To this end, first a surface matching process is used to find correspondences between these surfaces and the nodes of the mesh defining the internal and external surfaces in the model. Using these correspondences, a simple elastic transformation is applied to deform the complete mesh (solved as a linear perturbation problem using the commercial software ABAQUS, DSS, Providence, USA). Thus, an automatic procedure can be followed to construct finite element models of each patient in a matter of seconds. An example of this mesh adaptation can be seen in Fig. 4.

\subsection{Tissue Characterization}

Laboratory experiments were performed to characterise the two main tissues in the trachea: cartilage and muscle. There is no possibility of taking samples of internal membrane out of the trachea, but its structure is similar to the muscular membrane, so, its mechanical behaviour will be assumed to be the same. A blinded controlled trial on 20 patients was performed as follows. An experimental study consisting of an extension test 67 (up to 5\% deformation) of the cartilage was used to determine its elastic behaviour. A Neo-Hookean 8 curve regression was fitted to the experimental results, yielding to a value of $\mathrm{C}=0.56 \mathrm{MPa}$ for the stiffness of the cartilage. In order to determine the anisotropy of the material, histology was performed on several cuts over various tracheal rings. These observations allowed to confirm that tracheal cartilage is a material in which collagen fibres are randomly distributed.

Regarding the tracheal muscle, histology showed that it consists of two families of orthogonal fibres. In order to optimise computational time, we opted for building two different models, based on traction experiments of transversal and longitudinal tissue cuts. A Neo-Hookean material was assumed, and validated by curve fitting on the extension curves. The resulting parameters were $\mathrm{C}=0.032715 \mathrm{MPa}$ for the longitudinal and $\mathrm{C}=0.008984 \mathrm{MPa}$ for the transversal directions. For simulations of swallowing movement the longitudinal model was used, as this is the predominant direction of the movement. Conversely, the transversal model was used for simulations of sneezing movements.

Finally, the tracheal prosthesis was assumed to be elastic, since it is usually made of silicone.

\subsection{Simulation of Swallowing Movement}

Fluoroscopic image sequences were used to build a realistic model of the physiological swallowing movement. Several images were available, portraying both normal and pathological movement patterns, including explorations of patients after the implantation of endoprostheses. We adopted the movement of a healthy subject as the base for our simulations, as this is the movement that ideally would be recovered after the intervention. Further, it has been clinically seen that the movement of the trachea among different patients is not very different, because the glottis has to close to let the food going through the oesophagus.

Key anatomical landmarks (e.g. crycoid) are identified and tracked in the fluoroscopic images, and the resulting displacement fields are taken to the finite element 
analysis using correspondences on the deformed mesh. The finite element simulation, incorporating the models for the trachea and the implant, and reproducing the established movement pattern, was performed using ABAQUS.

\section{Results}

The framework was tested on data from 2 patients that underwent tracheal surgery. For each patient, two simulations were run, one on the data prior to the intervention and without incorporating the implant, and another one including the implant as positioned by the clinical expert using our tools. For the first patient (62 y.o., male, 83 $\mathrm{kg}$ ), we obtained a global displacement of the thyroid cartilage of $27.89 \mathrm{~mm}$, which is within the normal physiological range of $20-35 \mathrm{~mm}$, and a maximum principal tension of $0.487 \mathrm{MPa}$, when simulating without implant. Running the same simulation including the prosthesis led to a maximum displacement of $5.69 \mathrm{~mm}$ and a maximum principal tension of $0.61 \mathrm{MPa}$, meaning that the movement was reduced to only $20.4 \%$ of the original one, but the tension was 1.25 times higher.

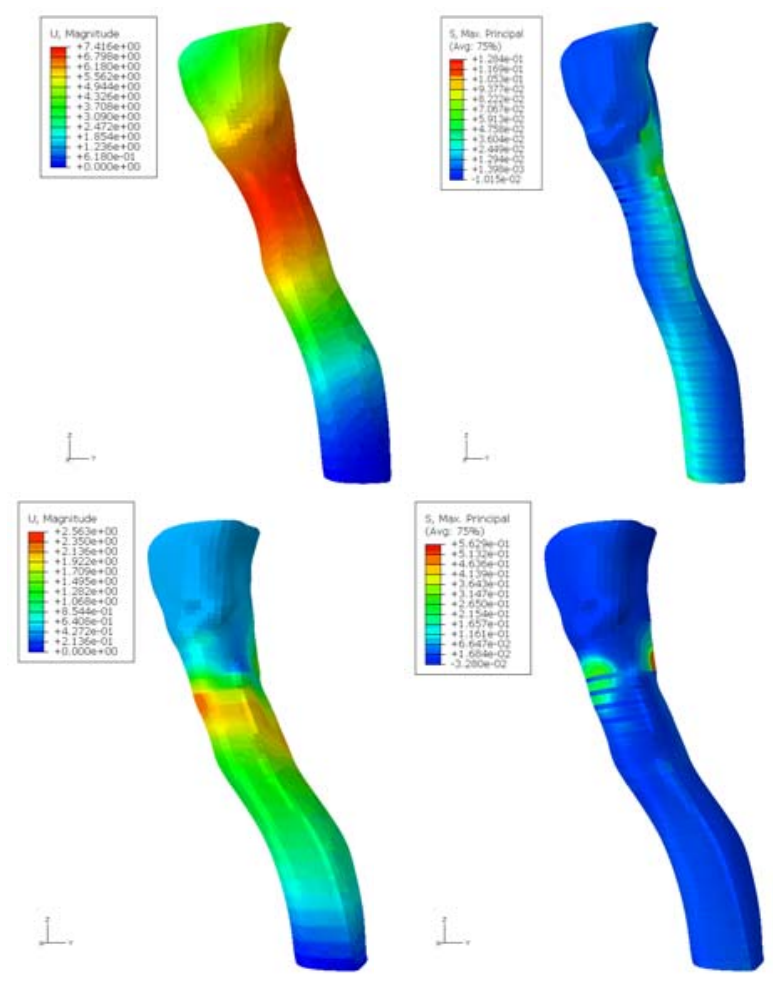

Fig. 5. Simulation of swallowing movement for patient 2, first without the implant (top row) and then simulating the implantation and the interaction with the tracheal wall (bottom row). For each case, we show the norm of the displacement (left) and the maximum tension (right). 
For the second patient ( 28 y.o., male, $75 \mathrm{~kg}$ ), the simulation without implant yielded a displacement of the reference cartilage of $7.41 \mathrm{~mm}$, with a maximum principal tension of $0.128 \mathrm{MPa}$. Incorporating the implant we obtained a displacement of $2.56 \mathrm{~mm}$ and a maximum principal tension of $0.563 \mathrm{MPa}$ (figure 5).

The results support the clinical hypothesis that tracheal implants lead to a loss of roughly $50 \%$ of the ability to swallow, and generate a damage (due to the increase in tension around the edges of the implant) that leads to the creation of granulomas in the tracheal wall 9.

\section{Conclusions}

We have developed a software for surgical planning of tracheal interventions, combining image segmentation, a database of virtual models of implants, and patientspecific biomechanical simulations of physiological movements to predict implant performance. Laboratory experiments were performed to characterise the tissue elements in the model, and adaptation of a detailed finite element mesh to fit the patient data was implemented. The method was tested on data from 2 patients, and results are clinically sound.

Several issues remain to be further explored. In future work, we plan to continue on the evaluation of the method an quantitatively evaluate its performance. That is, we will define clinical surrogates that can be measured from post-operative data and compared to the simulations. This is work in progress.

On the methodological side, one of the issues to be refined is the establishment of suitable movement models for different physiological processes. In addition to swallowing, sneezing movement simulations are underway. Further, movement models are currently obtained from fluoroscopic data of one patient (or normal subject). One of the approaches we are exploring is the construction of a parametric movement model, or even a statistical 4D model, such as the ones built for breathing movements 10 .

We believe that the results presented in this paper are promising and contribute to the development of novel therapies based on patient-specific virtual models including physiological simulations, which are bound to replace current ad-hoc surgical planning procedures and lead to principled evidence-based patient care.

Acknowledgments. This work was in part supported by Grant PI07/90023 of the Instituto de Salud Carlos III, Spain.

\section{References}

1. Smuk, P., Tiberiu, E., Evron, S., Roth, Y., Katz, J.: A brief history of tracheostomy and tracheal intubation, from the bronze age to the space age. Intensive Care Med. 34, 222-228 (2008)

2. Manniesing, R., Velthuis, B.K., van Leeuwen, M.S., van der Schaaf, I.C., van Laar, P.J., Niessen, W.J.: Level set based cerebral vasculature segmentation and diameter quantification in CT angiography. Medical image analysis 10(2), 200-214 (2006)

3. Perona, P., Malik, J.: Scale-space and edge detection using anisotropic diffusion. IEEE Transactions on Pattern Analysis and Machine Intelligence 12(7), 629-639 (1990) 
4. Chang, Y.L., Li, X.: Adaptive image region-growing. IEEE Transactions on Image Processing 3(6), 868-872 (1994)

5. Gonzalez, R.C., Woods, R.E.: Digital Image Processing, 2nd edn. Pearson, London (2001)

6. Teng, Z., Ochoa, I., Bea, J.A., Doblaré, M.: Theoretical and experimental studies on the nonlinear mechanical property of the tracheal cartilage. In: Procs. of 29th International Conference of the IEEE Engineering in Medicine and Biology, pp. 1058-1061 (2007)

7. Teng, Z., Ochoa, I., Li, Z., Lin, Y., Rodríguez, J., Bea, J., Doblaré, M.: Nonlinear mechanical property of tracheal cartilage: A theoretical and experimental study. Journal of Biomechanics 41(9), 1995-2002 (2008)

8. Holzapfel, G.A., Gasser, T.C., Ogden, R.W.: A new constitutive framework for arterial wall mechanics and a comparative study of material models. Journal of Elasticity $61,1-48$ (2000)

9. Noppen, M., Stratakos, G., D’Haese, J., Meysman, M., Vinken, W.: Removal of covered self-expandable metallic airway stents in benign disorders: indications, technique, and outcomes. Chest 127(2), 482-487 (2005)

10. von Siebenthal, M., Székely, G., Lomax, A., Cattin, P.C.: Inter-subject modelling of liver deformation during radiation therapy. In: Ayache, N., Ourselin, S., Maeder, A. (eds.) MICCAI 2007, Part I. LNCS, vol. 4791, pp. 659-666. Springer, Heidelberg (2007) 